\title{
Survival after Aortic Valve Replacement for Aortic Regurgitation: Prediction from Preoperative Contractility Measurement
}

\author{
Jeffrey S. Borer ${ }^{a}$ Phyllis G. Supino ${ }^{a}$ Edmund McM. Herrold ${ }^{a}$ \\ Antony Innasimuthu $^{\mathrm{a}}$ Clare Hochreiter ${ }^{\mathrm{b}}$ Karl Krieger ${ }^{\mathrm{b}}$ Leonard N. Girardi ${ }^{\mathrm{b}}$ \\ O. Wayne Isom ${ }^{b}$ \\ ${ }^{a}$ Division of Cardiovascular Medicine and The Howard Gilman Institute for Heart Valve Disease, State University of \\ New York Downstate Medical Center, Brooklyn, NY, USA; ${ }^{b}$ Department of Cardiothoracic Surgery, Weill Medical \\ College of Cornell University, New York, NY, USA
}

\author{
Keywords \\ Valvular heart disease $\cdot$ Exercise testing $\cdot$ Radionuclide \\ cineangiography
}

\begin{abstract}
Background: Noninvasive measurement of myocardial contractility (end-systolic wall stress-adjusted change in left ventricular ejection fraction from rest to exercise [ $\triangle \mathrm{LVEF}-$ $\triangle E S S]$ ) predicts heart failure, subnormal $L E_{\text {rest }}$, and sudden death in asymptomatic patients with chronic severe aortic regurgitation (AR). Here we assess the relation of preoperative $\triangle \mathrm{LVEF}-\triangle \mathrm{ESS}$ to survival after aortic valve replacement (AVR). Methods: Patients who underwent AVR for chronic, isolated, pure severe AR $(n=66)$ were followed for $13.0 \pm 6.4$ event-free years. Preoperative $\triangle \mathrm{LVEF}-\triangle \mathrm{ESS}$ (from combined echocardiographic and radionuclide cineangiographic data) enabled cohort stratification into 3 terciles ( -1 to $-11 \%$ [normal or mild] contractility deficit, -12 to $-16 \%$ [moderate], and $\leq-17 \%$ [severe], identical with segregation
\end{abstract}

in our earlier study) to relate preoperative contractility to postoperative survival and to age- and gender-matched US census data. Results: Since AVR, 22 patients died (average annual risk [AAR] for all-cause mortality for the entire cohort $=3.15 \%)$. Preoperative $\triangle \mathrm{LVEF}-\triangle \mathrm{ESS}$ predicted postoperative survival ( $p=0.009$, log rank test). By contractility terciles, all-cause AARs were $1.44,2.58$, and $6.40 \%$. Survival was lower than among US census comparators $(p<0.02)$, but the "mild" tercile was indistinguishable from census data ( $p=$ $n s)$. By multivariable Cox regression, survival prediction by pre-AVR $\triangle$ LVEF - $\triangle E S S$ was independent of, and superior to, prediction by age at surgery, gender, preoperative functional class, $\mathrm{LVEF}_{\text {rest, }} \mathrm{LVEF}_{\text {exercise, }}$ change in $\mathrm{LVEF}_{\text {rest }}$ to exercise, and LV diastolic or systolic dimensions ( $p \leq 0.01$, pre-AVR $\triangle \mathrm{LVEF}-\triangle \mathrm{ESS}$ vs. other covariates). Conclusion: In severe AR, preoperative contractility predicts post-AVR survival and may be prognostically superior to clinical, geometric and performance descriptors, potentially impacting on patient selection for surgery.

(c) 2018 The Author(s) Published by S. Karger AG, Basel

\begin{tabular}{ll}
\hline KARGER & $\begin{array}{l}\text { @ 2018 The Author(s) } \\
\text { Published by S. Karger AG, Basel Openger }\end{array}$ \\
E-Mail karger@karger.com & This article is licensed under the Creative Commons Attribution- \\
www.karger.com/crd & $\begin{array}{l}\text { NonCommercial-NoDerivatives 4.0 International License (CC BY- } \\
\text { NC-ND) (http://www.karger.com/Services/OpenAccessLicense). } \\
\text { Usage and distribution for commercial purposes as well as any dis- } \\
\text { tribution of modified material requires written permission. }\end{array}$
\end{tabular}

Jeffrey S. Borer, MD

State University of New York Downstate Medical Center 47 East 88th Street

New York, NY 10128-1152 (USA)

E-Mail jsborer1@gmail.com 


\section{Introduction}

When severe, aortic regurgitation (AR) gradually leads to irreversible left ventricular (LV) dysfunction, heart failure (HF), and death. Indeed, sudden death occurs in asymptomatic patients when intrinsic myocardial dysfunction is severe, even if $L V$ ejection fraction $(\mathrm{EF})$ is normal [1]. After aortic valve replacement (AVR) for AR, patients who were symptomatic before AVR manifest symptom reduction and, probably, better survival $[2,3]$ than expected from the natural progression of the disease. Myocardial damage from exogenous mechanical overloads mediates debility and death in AR. Therefore, AVR is appropriate when irreversible dysfunction is imminent [4]. In support of this strategy, survival after AVR is poorer when LVEF is subnormal than when normal [5], even if symptoms have not yet developed [6]. Moreover, LVEF gradually falls in patients with AR $[7,8]$, postoperative survival deteriorates as preoperative LVEF falls progressively below normal [9], and survival is affected independently by LVEF and by symptoms [9]. Therefore, delaying AVR until symptom onset in the asymptomatic patient is likely to compromise long-term survival [4]. However, it is difficult to know when irreversible dysfunction or symptoms are imminent. Moreover, evidence for commonly employed objective predictors of postAVR prognosis is relatively scant $[10,11]$. Randomized trials to define optimal timing of surgery have not been performed. Objective criteria for surgery generally are identified from comparison of matched observational cohorts that have and have not undergone AVR. Several empirically useful measures of LV size and EF thus have been accepted as indications for surgery even among asymptomatic patients [4]. However, when symptoms are absent and LVEF is normal at rest, earlier work by us [1] and others [12] suggests that development of clinically evident HF [13], subnormal LVEF at rest or sudden death in the absence of AVR are best predicted by intrinsic myocardial contractility $[1,12]$, which can be measured noninvasively [1]. Though suggested by small studies usually admixing several different valve lesions [14-17], the post-AVR prognostic utility of preoperative contractility is less well defined. Therefore, among an established cohort of patients with AR who underwent AVR, we aimed to relate postoperative survival to preoperative contractility and to compare the predictive value of this measure with previously established risk predictors $[1,8$, $18,19]$. In addition, we compared survival rates with those derived from contemporaneous age- and gendermatched US census data.

Survival after Aortic Valve Replacement for Aortic Regurgitation

\section{Methods}

\section{Patient Selection}

The study population was drawn from our prospective assessment of the natural history of regurgitant valve diseases and their predictors, ongoing for 30 years. Study details, including entry criteria for patients, have been described previously [8, 20-22]. At preoperative testing, all patients manifested hemodynamically severe AR confirmed at cardiac catheterization [23,24], without prior AVR, clinically evident coronary artery disease (documented myocardial infarction [MI] or angina) or hemodynamically important coronary obstructions at catheterization if coronary angiography was performed prior to AVR. At the time of analysis, 120 members of the cohort satisfied these criteria. Patients were then excluded if they had cardiac surgery in addition to AVR (10 patients), pre-AVR transvalvular systolic gradient $>10 \mathrm{~mm} \mathrm{Hg}$ or more than mild aortic stenosis by Doppler echocardiography ( 9 patients), more than mild mitral regurgitation (2 patients), or if they lacked preoperative echocardiography (8 patients) or preoperative radionuclide cineangiography (4 patients), or if $>1$ day separated echocardiogram and radionuclide cineangiogram (2 patients), or if $>1$ year separated AVR and preoperative testing (3 patients), or combinations of these criteria (16 patients). The 66 patients remaining after these exclusions comprise the study cohort. For this analysis, evaluation began at the pre-AVR index study which, as above, invariably occurred $<1$ year prior to surgery (average $2.7 \pm 2.9$ months, range $0.03-10.77$ ). Pre-AVR assessment comprised clinical evaluation plus several objective tests, including echocardiography at rest and radionuclide cineangiography at rest and during exercise as described previously [1, 14, 20, 22, 25]. Medical therapy and decisions for surgery were not influenced by the study protocol.

\section{Assessment of Preoperative Contractility}

Prior to AVR, M-mode and 2-dimensional echocardiography were performed as previously described [22-24]. American Society of Echocardiography recommendations [22] were followed for M-mode measurements of end-diastolic and end-systolic LV wall thicknesses, LV internal dimensions at diastole (LVIDd) and systole (LVIDs), and left atrial dimensions. LV mass was calculated as previously described [14]. End-systolic stress (ESS) at rest was defined using the method of Reichek et al. [13]: ESS $=(0.334 \times$ SBP $\times$ LVIDs) / (PWTs [1 + PWTs/LVIDs]), where SBP is systolic blood pressure and PWTs is posterior wall thickness in systole. LV end-systolic volume and LV end-diastolic volume were determined from LVIDs and LVIDd as per Teichholz et al. [26].

Radionuclide cineangiography was performed at a supine position at rest and during maximal, symptom-limited bicycle exercise using an electrocardiographically gated equilibrium method analogous to our earlier description [27, 28]. Red blood cells were technetium-labeled using a standard in vivo method involving intravenous injection of stannous pyrophosphate and $15-25 \mathrm{mCi}$ of technetium-99m. Our exercise protocol begins at a load of $25 \mathrm{~W}$, generally increasing by $25 \mathrm{~W}$ at 2-min intervals until limited by fatigue, dyspnea or hemodynamically important arrhythmias. Exercise LVEF is defined at peak exercise, generally involving at least the last $90 \mathrm{~s}$ of data collection. LVEF is determined using an emission (count)-based approach analogous to our earlier reports [2729], validated by comparison with contrast angiography at rest and exercise [29]. 
ESS during exercise was based on the assumption of conservation of mass from rest to exercise; SBP during exercise (cuff sphygmomanometry) was applied to resting echocardiographic wall thicknesses and chamber dimensions and modified from exerciseinduced changes in LV chamber volumes from radionuclide cineangiograms, as previously described [1]. Logistic difficulties associated with nuclear camera positioning precluded concurrent performance of echocardiography and radionuclide cineangiography. Data for these calculations were from studies usually performed sequentially during the same visit.

Because logarithmic transformation of ESS improves prediction of LV chamber function [30], each of 3 indices (LVEF $_{\text {rest }}$ normalized for $\mathrm{ESS}_{\text {rest }}, \mathrm{LVEF}_{\text {exercise }}$ normalized for $\mathrm{ESS}_{\text {exercise }}$ and $\triangle \mathrm{LVEF}_{\text {rest to exercise }}$ normalized for $\triangle \mathrm{ESS}_{\text {rest to exercise }}$ ) was defined using the natural log of ESS; the relation between the LVEF and ESS variables was defined by least squares linear regression. Studies in 26 normal subjects were used in regression equations to define the relations between rest, exercise and $\triangle \mathrm{LVEF}$ and the corresponding ESS variable; from these, the average value of each LVEF variable expected to be associated with a specific value of ESS was defined for normal subjects, as previously reported, for functional stratification by echocardiography $[31,32]$ alone and combined with radionuclide cineangiography [33]. The LVEF variable empirically measured for each patient was then subtracted from the LVEF variable expected in a normal subject at the same ESS. The difference, the $\triangle \mathrm{LVEF}-\triangle \mathrm{ESS}$ (or "LVEF-ESS index") variable, was used in statistical analyses; a negative value indicates a "contractility deficit." By prespecification, patients were subdivided into the 3 contractility terciles found to define prognostically important differences in our earlier publication [1]. In the current analysis, patients in these prespecified subgroupings serendipitously also formed statistically valid terciles; thus, the current study population is divided into no/mild ( $\triangle \mathrm{LVEF}-\Delta \mathrm{ESS}=-1$ to $-11 \%)$, moderate $(-12$ to $-16 \%)$ and severe $(\leq-17 \%)$ contractility deficit terciles.

\section{Follow-Up and Analysis}

Clinical follow-up continued annually after AVR by direct patient evaluation, telephone interview or review of primary physician charts until death, subsequent valve surgery, or until close of data collection in December 2011. The vital status of patients who were lost to follow-up was confirmed via a search of the Pension Benefit Information Participant Research Services Death Audit [34] database. Average event-free follow-up was $13.0 \pm 6.4$ years (range 0.6-26.0).

Kaplan-Meier product limit estimate curves were compared by $\log$ rank testing to provide univariable assessment of the relation of preoperative contractility deficit to post-AVR death (all causes) or to cardiovascular death (including sudden deaths and deaths due to HF, MI, stroke, endocarditis, and perioperative cardiac tamponade). These events were assessed as statistical terciles with test for linear trend. The one-sample log rank test [35] was used to compare survival in the entire cohort and its contractility-defined subgroups with the survival of age- and gender-matched comparators from the US census. Follow-up of patients who underwent repeat valvular surgery or other invasive therapies (e.g., defibrillator implantation) was censored as alive at the time of occurrence. A series of forward conditional multivariable Cox proportional hazards models (entry and removal thresholds, $p<0.05$ and $p<$ 0.10 , respectively) was constructed to determine the independent predictive value of preoperative contractility deficit for mortality
Table 1. Baseline characteristics of the study group $(n=66)$

\begin{tabular}{lc}
\hline Age, years & $49.15 \pm 15.28$ \\
Men & $57(86)$ \\
LVEF, \% & $46.61 \pm 11.11$ \\
FS, $\%$ & $26.69 \pm 0.069$ \\
LVIDd, cm & $7.45 \pm 1.03$ \\
LVIDs, cm & $5.50 \pm 1.10$ \\
LVM,$g$ & $402.19 \pm 114.53$ \\
NYHA functional class & \\
I & $25(38)$ \\
II & $23(35)$ \\
III & $16(24)$ \\
IV & $2(3)$ \\
Etiology & \\
Idiopathic/unknown & $15(23)$ \\
Idiopathic root dilatation & $14(21)$ \\
Rheumatic heart disease & $13(20)$ \\
Congenital abnormalities & $13(20)$ \\
Infective endocarditis & $4(6)$ \\
Degenerative and others & $4(6)$ \\
Connective tissue disease & $3(4)$ \\
Artificial mechanical prostheses & $33(50)$ \\
Preoperative cardiac medications & \\
None & $20(30)$ \\
Digoxin only & $6(9)$ \\
ACE inhibitor only & $1(2)$ \\
Alpha blocker only & $1(2)$ \\
Anti-arrhythmic only & $1(2)$ \\
Diuretic only & $1(2)$ \\
Combination therapy & $36(55)$ \\
\hline
\end{tabular}

Figures are means \pm SD or number of patients with percentages in parentheses. SD, standard deviation; LVEF, left ventricular ejection fraction; FS, fractional shortening; LVIDd, left ventricular internal dimension at diastole; LVIDs, left ventricular internal dimension at systole; LVM, LV mass; NYHA, New York Heart Association; ACE, angiotensin-converting enzyme.

prediction versus selected preoperative clinical risk factors, hemodynamic variables, and functional and other descriptors measured during preoperative radionuclide study or echocardiography versus intraoperative variables. To facilitate interpretation, variables entered into each Cox model were partitioned according to the same segregation values used for univariate analyses. The critical alpha level for defining statistical significance both in univariable and multivariable analysis was 0.05 . All data were analyzed using SPSS v16.0 (Chicago, IL, USA).

\section{Results}

\section{Baseline Characteristics}

Clinical characteristics and objective measures of LV size and performance at index study are presented in $\mathrm{Ta}$ ble 1 . Mean age was $49 \pm 15$ years; subjects were predom- 

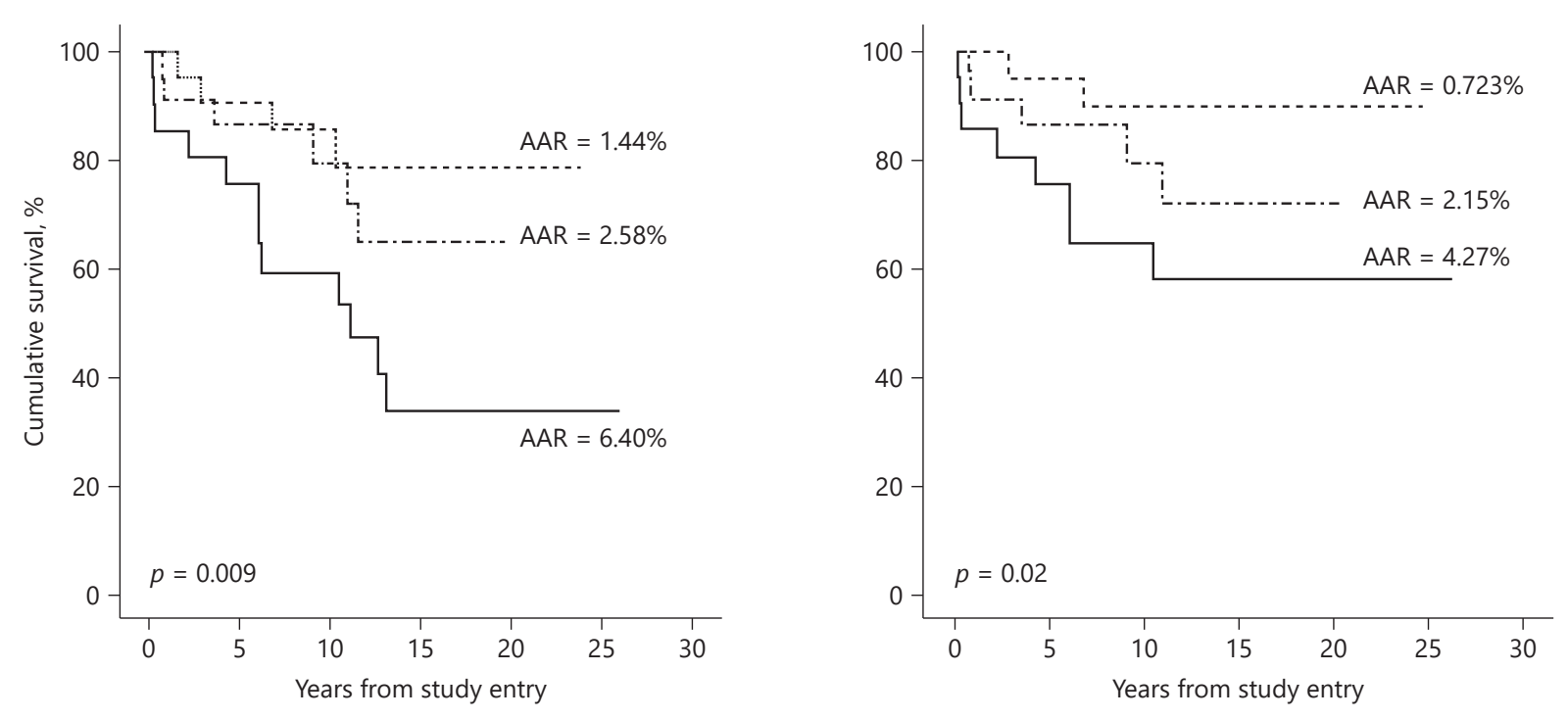

Fig. 1. Relationship of the $\triangle \mathrm{LVEF}-\triangle \mathrm{ESS}$ index at study entry (the time of the pre-aortic valve replacement [AVR] assessment) to cumulative survival (based on all-cause death and cardiovascular death) after AVR $(n=66)$. The left panel depicts results of all-cause death. The right panel depicts results of cardiovascular death (for definition, see text). In both panels, the dotted line represents the no/mild contractility deficit subgroup $(n=22)$, the dashed line represents the moderate contractility deficit subgroup $(n=21)$, and the solid line represents the severe contractility deficit subgroup $(n=23)$. AAR, average annual risk.

inantly male; AR etiology was predominantly idiopathic. Pre-AVR, most patients were taking digoxin chronically, either alone or in combination with other cardiac therapy. Artificial mechanical prostheses were employed in half the patients. Since hemodynamically important CAD at preoperative catheterization was a basis for exclusion from analysis, no patient in this series underwent concomitant coronary artery bypass grafting.

\section{Events during Follow-Up}

During follow-up, 22 patients died without intercurrent operation, including 15 cardiovascular deaths: sudden ( 6 patients), HF ( 3 patients), stroke (3 patients), MI (1 patient), endocarditis (1 patient), perioperative cardiac tamponade (1 patient); 7 died of putative noncardiovascular cause: liver cancer, prostate cancer, cytomegaloviral pneumonia, dementia-related aspiration, lupus-related kidney failure, HIV-positive acute liver failure, suicide (1 patient each); 17 patients were censored from further follow-up after subsequent cardiac surgery: AVR (10 patients), mitral valve replacement/repair (MVR/r, 2 patients), combined AVR and MVR/r (3 patients), combined MVR and tricuspid valve repair (1 patient), and pacemaker implantation (1 patient). Av- erage annual risk (AAR) of all causes of death among the entire cohort was 3.15\% (AAR of cardiovascular death was $2.15 \%$ ).

\section{Survival Prediction}

By univariable analysis, pre-AVR $\triangle \mathrm{LVEF}-\triangle \mathrm{ESS}$ (contractility) significantly predicted postoperative survival. When this measure fell within our prespecified no/ mild deficit tercile (22 patients), AAR after AVR was $1.44 \%$; for the moderate deficit tercile (23 patients), AAR was $2.58 \%$; for the severe deficit tercile (21 patients), AAR was $6.40 \%$ ( $p=0.009$, Fig. 1). Results were parallel when analysis was restricted to cardiovascular death: AAR = $0.72,2.15$, and $4.27 \%$ (no/mild, moderate, and severe terciles, respectively, $p=0.02$, Fig. 1 ).

For the entire study cohort, freedom from all causes of death was less than in the age- and sex-matched US census data ( $p<0.02$, Fig. 2$)$. However, for the no/mild contractility deficit tercile, survival was indistinguishable from the census comparator ( $p=$ ns, Fig. 2).

By multivariable Cox regression analysis, the predictive value of low pre-AVR $\triangle \mathrm{LVEF}-\triangle \mathrm{ESS}$ for all-cause mortality was independent of, and superior to, mortality prediction by age, gender, NYHA functional class, cross- 

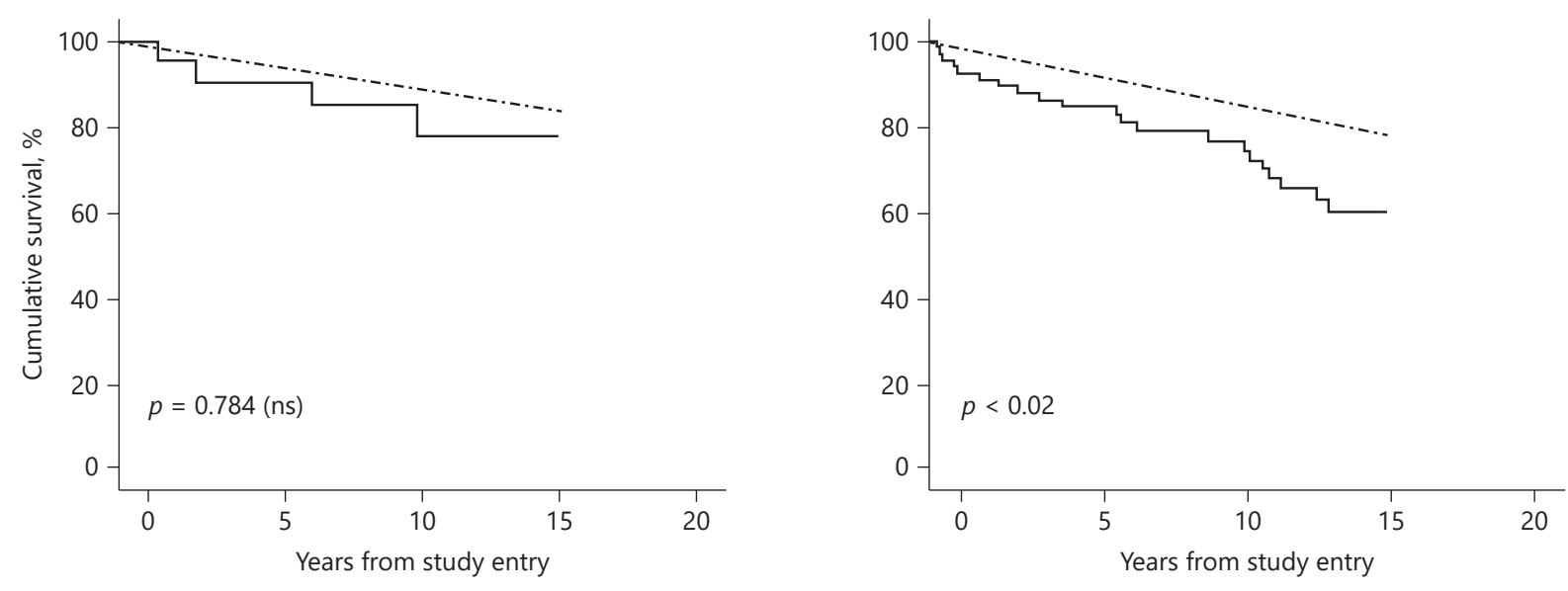

Fig. 2. Survival of AVR cohort versus gender- and age-matched US census comparators. The left panel depicts members of the cohort in the no/mild contractility deficit subgroup $(n=22)$ versus US census comparators. The solid line represents the no/mild contractility deficit subgroup; the dashed line represents the US census comparators. The right panel depicts all members of the cohort $(n=66)$ versus US comparators. The solid line represents the cohort; the dashed line represents the US census comparators.

clamp time, mechanical or bioprosthetic valve replacement, etiology, $\mathrm{LVEF}_{\text {rest }}, \mathrm{LVEF}_{\text {exercise, }} \triangle \mathrm{LVEF}_{\text {rest to exercise, }}$ LVIDs, LVIDd, fractional shortening, and preoperative medication use (digoxin, other cardiac medications, and combinations; ( $p \leq 0.01, \Delta \mathrm{LVEF}-\Delta \mathrm{ESS}$ vs. other covariates), entering first or exclusively in the final step of each model. Contractility was a similarly predictive value when analysis was limited to cardiovascular death $(p<0.04)$. Cox model analysis also revealed lack of interaction (ns [all interaction terms]) between pre-AVR $\triangle \mathrm{LVEF}-\triangle \mathrm{ESS}$ and NYHA functional class (I vs. II-IV or I-II vs. III-IV) or etiology (idiopathic, rheumatic, other) on prediction of post-AVR survival (freedom from all causes of death and from cardiovascular death); consistent with this finding, univariable analysis, undertaken post hoc within these subgroups, showed significantly $(p=0.002-0.046)$ or nominally $(p>0.10)$ poorer survival among patients in the severe contractility deficit tercile versus the other two terciles, mirroring patterns found for the entire cohort. Data were further analyzed by segregating patients into no/mild symptoms (functional classes I-II) and moderate/severe symptoms (functional classes III-IV). Allcause mortality and cardiovascular mortality based on $\triangle \mathrm{LVEF}-\triangle \mathrm{ESS}$ for both groups are shown in Figure 3. The small numbers of events in individual functional classes precluded meaningful comparison of one single class versus another.

\section{Discussion}

Our findings demonstrate that noninvasive measurement of myocardial contractility $(\triangle \mathrm{LVEF}-\triangle \mathrm{ESS})$ predicts late postoperative survival after AVR for AR. The predictive value of the contractility descriptor is independent of, and nominally superior to, previously employed clinical and objective indices in this population. This finding is consistent with our earlier report of contractility measurement to predict clinical outcome in patients with AR without prior AVR. The efficacy of preoperative contractility in predicting postoperative outcome is also consistent with earlier studies that have assessed performance descriptors, like LVEF and fractional shortening, which depend in part on contractility, and on the few relatively small studies in which contractility was measured by other methods in patients with a variety of valve diseases in which development of symptoms and "high-risk" LV dimensions (not mortality) were considered as outcome measures [36].

Other measures of LV dimensions and performance have been effective in prognostication in unoperated and operated patients. Subnormal LVEF is associated with relatively rapid progression to symptoms or death in asymptomatic unoperated patients with AR [6] and with relatively poor survival after AVR [6,37-39] compared with patients with normal LVEF $[3,17,29]$. Moreover, 


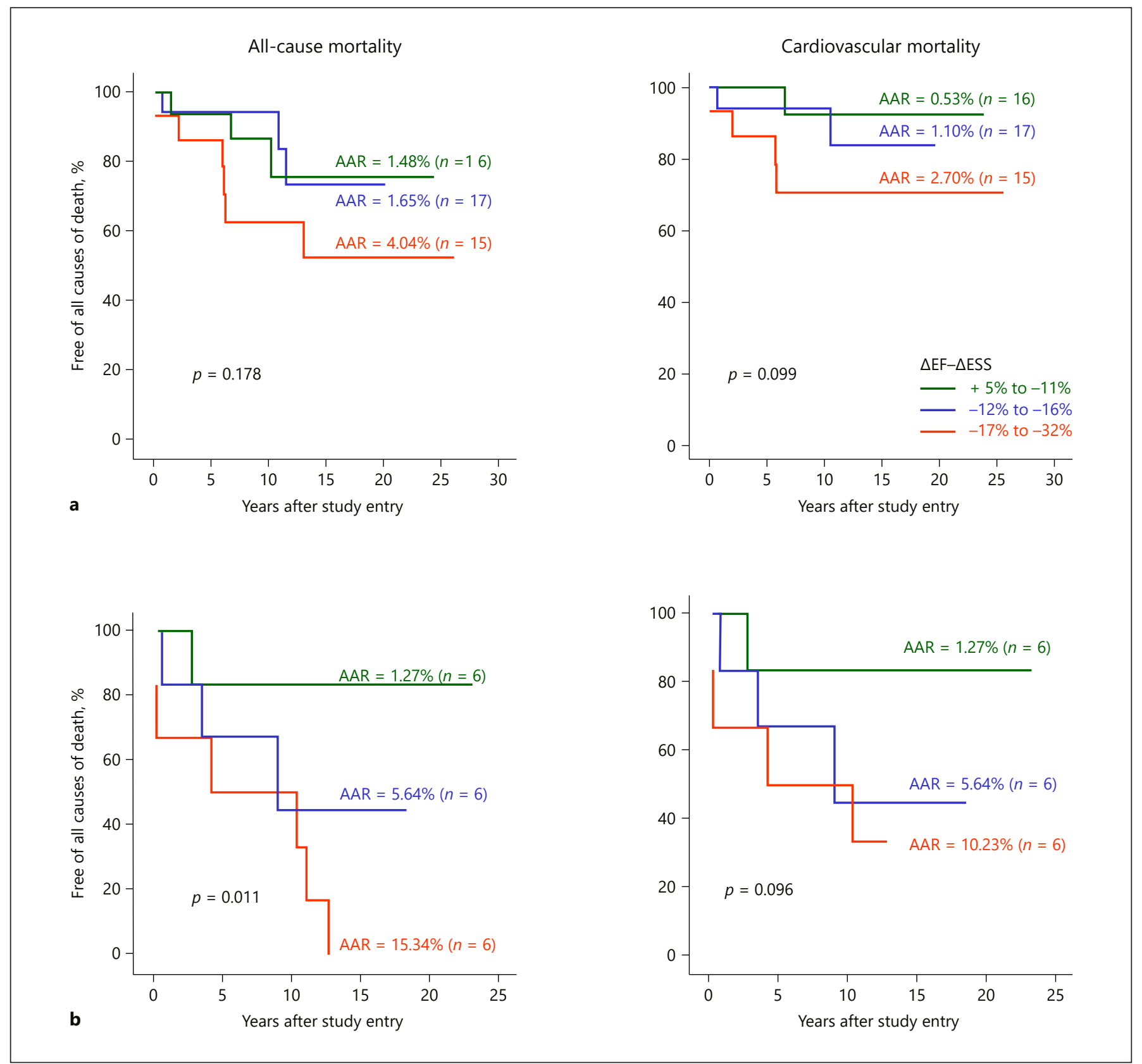

Fig. 3. Relationship of $\triangle \mathrm{LVEF}-\triangle \mathrm{ESS}$ and mortality in patients organized according to NYHA functional class. a Functional class I/II. b Functional class III/IV. In both $\mathbf{a}$ and $\mathbf{b}$, the left panel indicates all-cause mortality and the right panel presents cardiovascular mortality. The dotted lines represent patients with $\triangle \mathrm{LVEF}-\triangle \mathrm{ESS}$ of between +5 and $-11 \%$, dashed lines are patients with $\triangle \mathrm{LVEF}-\triangle \mathrm{ESS}$ of -12 to $-16 \%$ and the solid lines are patients with $\triangle \mathrm{LVEF}-\triangle \mathrm{ESS}$ of -17 to $-32 \%$.

though an increase in LVEF generally follows AVR (albeit not reaching the maximum until an interval exceeding 2 years [25]), patients with preoperatively subnormal LVEF may fail to improve after AVR. In both retrospec- tive [40-42] and prospective studies [37, 38, 43, 44], other LV performance descriptors, such as resting fractional shortening, exercise LVEF and $\triangle \mathrm{LVEF}_{\text {rest to exercise }}$ as well as LV dimensions, have predicted outcome after 
AVR. Small, older studies, though not focusing on survival, suggest that irreversible myocardial contractile dysfunction before AVR may presage persistent myocardial dysfunction after AVR. More recently, LV global longitudinal strain was found to be a prognosticator for survival after AVR in a homogeneous group of patients with preserved LVEF [45]. However, this does not take into consideration the effects of loading conditions and changes in loading conditions (rest $\rightarrow$ exercise) on the LVEF due to blood pressure, a deficiency not present in the current study. Nonetheless, these studies do support inclusion of a more precise assessment of LV function in determining the prognosis of patients with chronic severe AR. Previous studies further suggest that contractile function is superior as a predictor of irreversible postAVR myocardial dysfunction than preoperative LV dimensions or LVEF [15], supporting the likelihood of its utility for clinical outcome prediction. However, contractility has not been previously studied for prediction of survival after AVR for severe AR. Nonetheless, in the single prospective study in which it was evaluated [1], intrinsic myocardial contractility was superior to LVEF or dimension descriptors in predicting outcome in asymptomatic and minimally symptomatic unoperated patients with severe AR, suggesting its probable utility for post-AVR prediction. In theory, intrinsic myocardial contractility has advantages over performance and size descriptors in assessing clinical sequelae. Measures of contractility, as a group, are relatively load independent, unlike LVEF and dimensions [44]. The importance of LV loads in dissociating LVEF from contractility has been suggested by Scognamiglio et al. [46] who found that, among patients who underwent AVR with subnormal LVEF, 10 -year postoperative survival was $85 \pm 4 \%$ in hypertensive patients receiving pharmacological unloading treatment compared to $78 \pm 5 \%$ in patients not receiving such therapy, despite similar LVEF and LV diastolic and systolic volumes. Hence, myocardial contractility may be a more accurate reflection of myocardial status and subsequent clinical results than surrogates like LVEF or dimensions/volumes.

In an earlier era, onset of symptoms was generally employed as the primary indication for AVR in AR; symptoms remain an appropriate basis for AVR. AVR will reduce or relieve symptoms and probably will improve survival in the symptomatic patient. However, available data suggest that intrinsic myocardial dysfunction often precedes the onset of symptoms (and development of subnormal LVEF, a well-accepted indication for AVR). Thus, in theory, the contractility measure should be more useful in prognostication and patient selection than other criteria now employed. The data from this study support this hypothesis. Increasing emphasis has been placed on myocardial biology to compensate for the prognostic inadequacies of conventional size and performance descriptors in AR [47-50]; intrinsic contractility may prove an integrator of these various biological responses.

Inferences from observational series support consensus guidelines defining indications for AVR, but no randomized trials have assessed the life-prolonging efficacy of surgery. The result has been uncertainty as to appropriate bases for surgery, particularly in asymptomatic patients. However, as outcomes after conventional surgical AVR progressively improve, and as relatively less morbid percutaneous approaches for AVR in AR eventually are developed, benefit/risk considerations may favor earlier application of AVR than currently [51]. In the absence of randomized trials, the current findings, together with our earlier study of nonoperated patients (most of whom entered the study during approximately the same temporal interval as this AVR cohort and, thus, were studied contemporaneously for many years), suggest that noninvasive measurement of intrinsic contractility may be a particularly appropriate basis for optimizing selection of patients for surgery. Given the relatively poorer predictive value of conventional prognostic criteria, suggested by our data, the use of noninvasive contractility measures, like that one employed here, appears potentially useful.

\section{Study Limitations}

Our study is a longitudinal observational analysis and is subject to all possible limitations of such studies, including selection and referral biases. Approximately half our patients had idiopathic AR and one quarter had rheumatic AR. Findings from this study may be most applicable to these etiologic groups, though these and other etiologies represented in the sample did not differ substantially in outcome from the results as a whole. In addition, most of our patients were men. Thus, our results may not be optimally generalizable to women, who are more likely than men to develop patient-prosthesis mismatch post-AVR and, consequently, to manifest poor outcomes more frequently than men [52]. Nonetheless, the size of our population is consistent with others from which current guidelines have been drawn. 


\section{Conclusions}

Preoperative noninvasive myocardial contractility measurement effectively predicts post-AVR survival. Contractility measurement may be superior for such prediction to the presence or absence of symptoms or to measures of LV dimensions or performance. Since myocardial dysfunction generally occurs before symptom onset, contractility may be particularly useful in identifying asymptomatic patients for whom AVR is appropriate, though this hypothesis requires further study for rigorous support. Taken together with our earlier data using this same measure to predict outcome among unoperated patients with AR, which directly parallel the current findings, these results strongly suggest that noninvasive myocardial contractility measurement should be routinely employed in the evaluation of patients with AR.

\section{Acknowledgment}

During the performance of this work, Dr. Borer was supported in part as The Gladys and Roland Harriman Professor of Cardiovascular Medicine, Dr. Krieger as The Michel Bergerac Professor of Cardiothoracic Surgery, and Dr. Isom as The Terry Allen Kramer Professor of Cardiothoracic Surgery, Weill Cornell Medical College; in addition, this work was supported by grants from the National Heart Lung and Blood Institute, Bethesda, MD (RO1HL-26504, J. Borer, P.I.), The Howard Gilman Foundation, New York, N.Y., The Schiavone Family Foundation, White House Station, N.J., The Charles and Jean Brunie Foundation, Bronxville, N.Y., The David Margolis Foundation, New York, N.Y., The American Cardiovascular Research Foundation, New York, N.Y., The Irving A. Hansen Foundation, New York, N.Y., The Mary A.H. Rumsey Foundation, New York, N.Y., The Messinger Family Foundation, New York, N.Y., The Daniel and Elaine Sargent Charitable Trust, New York, N.Y., and by much appreciated gifts from Donna and William Acquavella, New York, N.Y., Maryjane Voute Arrigoni and the late William Voute, Bronxville, N.Y., Gerald Tanenbaum, New York, N.Y., and Suzanne and the late Stephen Weiss, Greenwich, CT.

\section{References}

1 Borer JS, Hochreiter C, Herrold EM, et al: Prediction of indications for valve replacement among asymptomatic or minimally symptomatic patients with chronic aortic regurgitation and normal left ventricular performance. Circulation 1998;97:525-534.

2 Kvidal P, Bergstrom R, Malm T, Stahle E: Long-term follow-up of morbidity and mortality after aortic valve replacement with a mechanical valve prosthesis. Eur Heart J 2000; 21:1099-1111.

3 Kvidal P, Bergstrom R, Horte LG, Stahle E: Observed and relative survival after aortic valve replacement. J Am Coll Cardiol 2000;35: 747-756.

4 Bonow RO, Carabello BA, Chatterjee K, et al: 2008 focused update incorporated into the ACC/AHA 2006 guidelines for the management of patients with valvular heart disease: a report of the American College of Cardiology/American Heart Association Task Force on Practice Guidelines (Writing Committee to Revise the 1998 Guidelines for the Management of Patients With Valvular Heart Disease): endorsed by the Society of Cardiovascular Anesthesiologists, Society for Cardiovascular Angiography and Interventions, and Society of Thoracic Surgeons. Circulation 2008;118:e523-e661.

5 Bonow RO: Radionuclide angiography in the management of asymptomatic aortic regurgitation. Circulation 1991;84(suppl 3):I296-I302.

6 Henry WL, Bonow RO, Rosing DR, Epstein SE: Observations on the optimum time for operative intervention for aortic regurgitation. II. Serial echocardiographic evaluation of asymptomatic patients. Circulation 1980; 61:484-492.
7 Borer JS, Herrold EM, Hochreiter CA, et al: Aortic regurgitation: selection of asymptomatic patients for valve surgery. Adv Cardiol 2002;39:74-85.

8 Bonow RO, Lakatos E, Maron BJ, Epstein SE: Serial long-term assessment of the natural history of asymptomatic patients with chronic aortic regurgitation and normal left ventricular systolic function. Circulation 1991; 84:1625-1635.

9 Chaliki HP, Mohty D, Avierinos JF, et al: Outcomes after aortic valve replacement in patients with severe aortic regurgitation and markedly reduced left ventricular function. Circulation 2002;106:2687-2693.

10 Hannan EL, Racz MJ, Jones RH, et al: Predictors of mortality for patients undergoing cardiac valve replacements in New York State. Ann Thorac Surg 2000;70:1212-1218.

11 He GW, Acuff TE, Ryan WH, et al: Aortic valve replacement: determinants of operative mortality. Ann Thorac Surg 1994;57:11401146.

12 Greves J, Rahimtoola SH, McAnulty JH, et al: Preoperative criteria predictive of late survival following valve replacement for severe aortic regurgitation. Am Heart J 1981;101:300308.

13 Reichek N, Wilson J, St John Sutton M, et al: Noninvasive determination of left ventricular end-systolic stress: validation of the method and initial application. Circulation 1982;65: 99-108.

14 Carabello BA, Nolan SP, McGuire LB: Assessment of preoperative left ventricular function in patients with mitral regurgitation: value of the end-systolic wall stress-end-systolic volume ratio. Circulation 1981;64:1212-1217.
15 Kumpuris AG, Quinones MA, Waggoner $\mathrm{AD}$, et al: Importance of preoperative hypertrophy, wall stress and end-systolic dimension as echocardiographic predictors of normalization of left ventricular dilatation after valve replacement in chronic aortic insufficiency. Am J Cardiol 1982;49:1091-1100.

16 Gaasch WH, Carroll JD, Levine HJ, Criscitiello MG: Chronic aortic regurgitation: prognostic value of left ventricular end-systolic dimension and end-diastolic radius/thickness ratio. J Am Coll Cardiol 1983;1:775-782.

17 Starling MR, Kirsh MM, Montgomery DG, Gross MD: Mechanisms for left ventricular systolic dysfunction in aortic regurgitation: importance for predicting the functional response to aortic valve replacement. J Am Coll Cardiol 1991;17:887-897.

18 Dujardin KS, Enriquez-Sarano M, Schaff HV Bailey KR, Seward JB, Tajik AJ: Mortality and morbidity of aortic regurgitation in clinical practice. A long-term follow-up study. Circulation 1999;99:1851-1857.

19 Bonow RO, Rosing DR, McIntosh CL, et al: The natural history of asymptomatic patients with aortic regurgitation and normal left ventricular function. Circulation 1983;68:509-517.

20 Roman MJ, Klein L, Devereux RB, et al: Reversal of left ventricular dilatation, hypertrophy, and dysfunction by valve replacement in aortic regurgitation. Am Heart J 1989;118: 553-563.

21 Roman MJ, Devereux RB, Niles NW, et al: Aortic root dilatation as a cause of isolated severe aortic regurgitation. Prevalence, clinical and echocardiographic patterns, and relation to left ventricular hypertrophy and function. Ann Intern Med 1987;106:800-807. 
22 Niles N, Borer JS, Kamen M, Hochreiter C, Devereux RB, Kligfield P: Preoperative left and right ventricular performance in combined aortic and mitral regurgitation and comparison with isolated aortic or mitral regurgitation. Am J Cardiol 1990;65:13721378.

23 Sahn DJ, DeMaria A, Kisslo J, Weyman A: Recommendations regarding quantitation in M-mode echocardiography: results of a survey of echocardiographic measurements. Circulation 1978;58:1072-1083.

24 Devereux RB, Alonso DR, Lutas EM, et al: Echocardiographic assessment of left ventricular hypertrophy: comparison to necropsy findings. Am J Cardiol 1986;57:450-458.

25 Borer JS, Herrold EM, Hochreiter C, et al: Natural history of left ventricular performance at rest and during exercise after aortic valve replacement for aortic regurgitation. Circulation 1991;84:III133-III139.

26 Teichholz LE, Kreulen T, Herman MV, Gorlin R: Problems in echocardiographic volume determinations: echocardiographic-angiographic correlations in the presence of absence of asynergy. Am J Cardiol 1976;37: 7-11.

27 Borer JS, Bacharach SL, Green MV, et al: Exercise-induced left ventricular dysfunction in symptomatic and asymptomatic patients with aortic regurgitation: assessment with radionuclide cineangiography. Am J Cardiol 1978; 42:351-357.

28 Borer JS, Bacharach SL, Green MV, Kent KM, Epstein SE, Johnston GS: Real-time radionuclide cineangiography in the noninvasive evaluation of global and regional left ventricular function at rest and during exercise in $\mathrm{pa}-$ tients with coronary-artery disease. N Engl J Med 1977;296:839-844.

29 Goldberg HL, Moses JW, Borer JS, et al: Exercise left ventriculography utilizing intravenous digital angiography. J Am Coll Cardiol 1983;2:1092-1098.

30 Lutas EM, Devereux RB, Reis G, et al: Increased cardiac performance in mild essential hypertension. Left ventricular mechanics. Hypertension 1985;7:979-988.

31 Hammond IW, Devereux RB, Alderman MH, Laragh JH: Relation of blood pressure and body build to left ventricular mass in normotensive and hypertensive employed adults. J Am Coll Cardiol 1988;12:996-1004.
32 Ganau A, Devereux RB, Atlas SA, et al: Plasma atrial natriuretic factor in essential hypertension: relation to cardiac size, function and systemic hemodynamics. J Am Coll Cardiol 1989;14:715-724; discussion 725-727.

33 Borer JS, Jason M, Devereux RB, Pickering T, Erle S, Laragh JH: Left ventricular performance in the hypertensive patient. Exercisemediated noninvasive separation of loading influences from intrinsic muscle dysfunction. Chest 1983;83:314-316.

34 http://pbinfo.com/death-audit-service (accessed February 20, 2012).

35 Berry G: The analysis of mortality by the subject-years method. Biometrics 1983;39:173184.

36 Percy RF, Miller AB, Conetta DA: Usefulness of left ventricular wall stress at rest and after exercise for outcome prediction in asymptomatic aortic regurgitation. Am Heart J 1993;125:151-155.

37 Carabello BA, Williams H, Gash AK, et al: Hemodynamic predictors of outcome in patients undergoing valve replacement. Circulation 1986;74:1309-1316.

38 Klodas E, Enriquez-Sarano M, Tajik AJ, Mullany CJ, Bailey KR, Seward JB: Aortic regurgitation complicated by extreme left ventricular dilation: long-term outcome after surgical correction. J Am Coll Cardiol 1996;27:670677.

39 Acar J, Michel PL, Luxereau P, et al: How to manage patients with severe left ventricular dysfunction and valvular regurgitation. J Heart Valve Dis 1996;5:421-429.

40 Cunha CL, Giuliani ER, Fuster V, Seward JB, Brandenburg RO, McGoon DC: Preoperative M-mode echocardiography as a predictor of surgical results in chronic aortic insufficiency. J Thorac Cardiovasc Surg 1980;79:256-265.

41 Forman R, Firth BG, Barnard MS: Prognostic significance of preoperative left ventricular ejection fraction and valve lesion in patients with aortic valve replacement. Am J Cardiol 1980;45:1120-1125.

42 Recke SH, Marienhagen J, Feistel H, Platsch G, Bock E, von der Emde J: Electrocardiographic characteristics indicating a risk of irreversibly impaired myocardial function in chronic aortic regurgitation. Int J Cardiol 1993;42:129-138.
43 Bonow RO, Picone AL, McIntosh CL, et al: Survival and functional results after valve replacement for aortic regurgitation from 1976 to 1983: impact of preoperative left ventricular function. Circulation 1985;72:1244-1256.

44 Bashore TM: Afterload reduction in chronic aortic regurgitation: it sure seems like a good idea. J Am Coll Cardiol 2005;45:1031-1033.

45 Alashi A, Mentias A, Abdallah A: Incremental prognostic utility of left ventricular global longitudinal strain in asymptomatic patients with significant chronic aortic regurgitation and preserved left ventricular ejection fraction. JACC Cardiovasc Imaging 2018; 11:673682

46 Scognamiglio R, Negut C, Palisi M, Fasoli G, Dalla-Volta S: Long-term survival and functional results after aortic valve replacement in asymptomatic patients with chronic severe aortic regurgitation and left ventricular dysfunction. J Am Coll Cardiol 2005;45:10251030.

47 Ozkan M, Baysan O, Erinc K, et al: Brain natriuretic peptide and the severity of aortic regurgitation: is there any correlation? J Int Med Res 2005;33:454-459.

48 Pizarro R, Bazzino OO, Oberti PF, et al: Prospective validation of the prognostic usefulness of B-type natriuretic peptide in asymptomatic patients with chronic severe aortic regurgitation. J Am Coll Cardiol 2011;58: 1705-1714.

49 Borer JS, Truter S, Herrold EM, et al: Myocardial fibrosis in chronic aortic regurgitation: molecular and cellular responses to volume overload. Circulation 2002;105:1837-1842.

50 Truter SL, Catanzaro DF, Supino PG, et al: Differential expression of matrix metalloproteinases and tissue inhibitors and extracellular matrix remodeling in aortic regurgitant hearts. Cardiology 2009;113:161-168.

51 Tornos P, Sambola A, Permanyer-Miralda G, Evangelista A, Gomez Z, Soler-Soler J: Longterm outcome of surgically treated aortic regurgitation: influence of guideline adherence toward early surgery. J Am Coll Cardiol 2006; 47:1012-1017.

52 Walther T, Rastan A, Falk V, et al: Patient prosthesis mismatch affects short- and longterm outcomes after aortic valve replacement. Eur J Cardiothorac Surg 2006;30:15-19. 